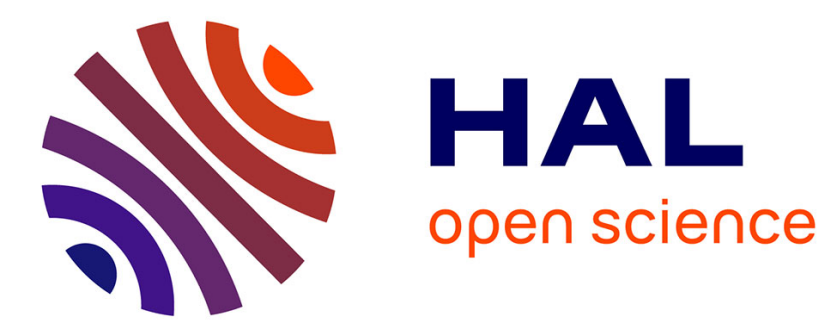

\title{
Path Following Control for an Eel-Like Robot
}

Lionel Lapierre, Bruno Jouvencel

\section{To cite this version:}

Lionel Lapierre, Bruno Jouvencel. Path Following Control for an Eel-Like Robot. OCEANS, Jul 2005, Brest, France. pp.460-465, 10.1109/OCEANSE.2005.1511759 . lirmm-00106474

\section{HAL Id: lirmm-00106474 https://hal-lirmm.ccsd.cnrs.fr/lirmm-00106474}

Submitted on 16 Oct 2006

HAL is a multi-disciplinary open access archive for the deposit and dissemination of scientific research documents, whether they are published or not. The documents may come from teaching and research institutions in France or abroad, or from public or private research centers.
L'archive ouverte pluridisciplinaire HAL, est destinée au dépôt et à la diffusion de documents scientifiques de niveau recherche, publiés ou non, émanant des établissements d'enseignement et de recherche français ou étrangers, des laboratoires publics ou privés. 


\section{Path Following Control for an Eel-like Robot}

\author{
Lionel Lapierre \\ LIRMM \\ Montpellier, France \\ Email: lapierre@lirmm.fr
}

\author{
Bruno Jouvencel \\ LIRMM \\ Montpellier, France \\ Email: jouvencel@lirmm.fr
}

\begin{abstract}
We investigate the problem of controlling the motion of an eel like-robot. Recent work has shown promising results for this type of systems, opening new issues in the field of efficient propulsion and high manoeuvrability systems. The motion planning is decoupled in the two subproblems of thrust generation and heading control. In this paper we investigate a new type of autonomous gait generation, explicitly controlling the local system curvatures. The solution is then coupled with a path following controller, using the virtual target principle. The controller design is based on Lyapunov techniques. Simulations illustrate the performances of the proposed solution.
\end{abstract}

\section{INTRODUCTION}

Since a long time, biologically inspired solutions have motivated a large number of robotic applications. In the domain of underwater robotics, the question of efficient and low consumption underwater propulsion, in conjunction with a high manoeuvrability is an active field of research. A whale tail is an 'unreachable' reference in terms of thrust efficiency; the lamprey is able of incredible maneuvers. The marine biologists have studied this type of locomotion, from the lamprey to the tuna fish, and have identified the body shape evolution to produce a thrust along the body axis. From a robotics point of view, this body shape evolution is a propagating wave (also called 'gait'), adjusting the signal phase according to the joint situation located on each vertebrae. From these results, a large number of interesting robotic applications have been performed in the last decades. The first question to be solved, concerning the model derivation of such a flexible system, is generally solved considering a highly coupled, non linear, hyper redundant model. The motion control problem is decoupled into three sub-problems:

- the gait generation,

- the control of the joint actuation in order to follow the previous reference,

- the adaptation of the gait parameters according to the system situation with respect to the main path the system has to reach and follow.

The gait generation is a crucial question in this problem. Indeed, a bad chosen gait will agitate the robot, without guarantying that the maximum number of the system elements (vertebrae) are involved in the movement, loosing by the way the desired efficiency, in terms of thrust and manoeuvrability.

The solutions of the literature propose some actuation gaits, directly controlling the joints as a trajectory tracker, or a kinematic joint control to follow the desired body shape ([1],

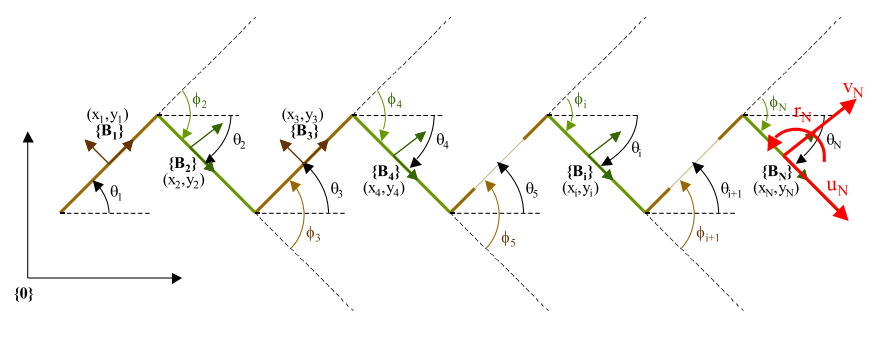

Fig. 1. Problem Pose

[3], [2]). The loop is closed on the gait parameters, adapting them in function of the system situation with respect to the main goal (path). An interresting optimal approach is described in [4]. Neural approach has been also tested, and promising results can be found in [5] and [6].

In this paper, we propose a method to drive the system according to a reference body curvature, which is not time dependant and that explicitly takes into account the system dynamics. The control is derived using Lyapunov theory and Backstepping techniques, in order to guarantee the system to asymptotically converge to the desired shape defined in function of the curvilinear abscissa of each joints on the reference to be followed. The reference parameters are then adjusted in order for the system to reach a desired path, at a desired forward velocity. The path following algorithm is using the 'virtual target' guidance principle.

The paper is organized as follow : chapter II describes the system modeling, chapter III introduces the control design method we are proposing and chapter IV indicates some simulation results to illustrate the performances of our solution.

\section{SySTEM MODELING}

We model the eel-like robot as a planar, serial chain of $N$ links of length $d_{i}$, mass $m_{i}$ and inertia $I_{i}$, where $i=1 \ldots N$. Referring to the figure 1, we define two groups of kinematic variables called configuration variables $\dot{q}$ and shape variable $\dot{s}$, denoted as :

$$
\begin{gathered}
\dot{q}=\left[u_{1} v_{1} r_{1} \dot{\phi}_{2} \dot{\phi}_{3} \ldots \dot{\phi}_{N-1} \dot{\phi}_{N}\right]^{T} \\
\dot{s}=\left[u_{2} v_{2} u_{3} v_{3} \ldots u_{N} v_{N}\right]^{T}
\end{gathered}
$$

where $u_{i}$ is the forward velocity of the link $i, v_{i}$ is the side-slip velocity, and $r_{i}$ the rotational velocity, expressed in the body axis $\left\{B_{i}\right\} . \phi_{i}$ is the relative angle between the link $i-1$ and $i$. Note that the variables $\phi_{i}$ can be called 
articular variables, and that the group $q$ defines a necessary and sufficient set to describe the system situation.

\section{A. Hydrodynamics Approximation}

We model each link of the eel-like robot as a cylinder of length $d_{i}$, radius $L_{i}$ and mass $m_{i}$. The significant hydrodynamical forces applied on the systems are 3 types:

- perpendicular drag effect : we assume that differential pressure only acts perpendicularly to the body. Note that this is the force that produces the system thrust. We consider the approximation used in [2].

$$
F_{i}^{v}=-\mu_{i} v_{i}
$$

where $F_{i}$ is the perpendicular drag force acting in the link $i$, moving with a side-slip velocity $v_{i} ; \mu_{i}$ is the perpendicular drag coefficient of the considered system element.

- Parallel drag effect : due to the streamlined nature of the body of the eel, the friction coefficient in the body direction is negligible with respect to the perpendicular direction ([2], [6]). Nevertheless, this effect is significant on the first link (also called head) for forward movement, and on the last segment for backward movement (tail).

$$
\begin{aligned}
& F_{1}^{u}=\left\{\begin{array}{ccc}
-\nu_{1} u_{1} & \text { if } & u_{1}>0 \\
0 & \text { if } & u_{1} \leq 0
\end{array}\right. \\
& F_{N}^{u}=\left\{\begin{array}{ccc}
-\nu_{N} u_{N} & \text { if } & u_{1}<0 \\
0 & \text { if } & u_{N} \geq 0 \\
F_{i}^{u} & =\text { for } i=2 \text { to } N-1
\end{array}\right.
\end{aligned}
$$

where $F_{1}^{u}$ and $F_{N}^{u}$ are the parallel drag effect acting on the head and the tail, respectively, $\nu_{i}, i=1, N$ are the parallel drag coefficient, and $u_{i}$ denotes the forward speed of the $i^{t h}$ element.

- Added mass : the added mass term is estimated considering the cylindrical shape of the system components. We refer to [7] for an approximation of this effect parameters. The inertial matrix for the $i^{\text {th }}$ element, expressed in the body frame $\left\{B_{i}\right\}$ is written as :

$$
M_{i}=\left[\begin{array}{ccc}
m_{i}^{x} & 0 & 0 \\
0 & m_{i}^{y} & 0 \\
0 & 0 & I^{i}
\end{array}\right]
$$

where $m_{i}^{x}$ and $m_{i}^{y}$ is the wet mass (intertial and added mass)in the $x$ and $y$ direction of the body axis $\left\{B_{i}\right\}$, and $I_{i}$ is the wet moment of inertial the $i^{\text {th }}$ element.

\section{B. Model Derivation}

Thanks to the variable definition in (1) the system Lagrangian is written.

$$
L=\frac{1}{2} \sum_{i=1}^{N} \dot{\eta}_{i}^{T} M_{i} \dot{\eta}
$$

where $\dot{\eta}_{i}=\left[u_{i} v_{i} r_{i}\right]$ denotes the $i^{\text {th }}$ element velocity expressed in the body frame $\left\{B_{i}\right\}$. The previous Lagrangian expression is rewritten as :

$$
L=\frac{1}{2} \dot{q}^{T} M^{1} \dot{q}+\frac{1}{2} \dot{s}^{T} M^{2} \dot{s}
$$

where

$$
M^{1}=\left[\begin{array}{cccccc}
m_{1}^{x} & 0 & 0 & 0 & \ldots & 0 \\
0 & m_{1}^{y} & 0 & 0 & \ldots & 0 \\
0 & 0 & a_{1} & a_{2} & \ldots & I_{N} \\
0 & 0 & a_{2} & a_{2} & \ldots & I_{N} \\
\ldots & \ldots & \ldots & \ldots & \ldots & \ldots \\
0 & 0 & I_{N} & I_{N} & \ldots & I_{N}
\end{array}\right]
$$

with $a_{i}=\sum_{k=i}^{N} I_{k}$ and

$$
M^{2}=\left[\begin{array}{ccccccc}
m_{2}^{x} & 0 & 0 & 0 & \ldots & 0 & 0 \\
0 & m_{2}^{y} & 0 & 0 & \ldots & 0 & 0 \\
0 & 0 & m_{3}^{x} & 0 & \ldots & 0 & 0 \\
0 & 0 & 0 & m_{3}^{y} & \ldots & 0 & 0 \\
\ldots & \ldots & \ldots & \ldots & \ldots & \ldots & \\
0 & 0 & 0 & 0 & \ldots & m_{N}^{x} & 0 \\
0 & 0 & 0 & 0 & \ldots & 0 & m_{N}^{y}
\end{array}\right]
$$

The consideration of the following kinematic relations in the body frames :

$$
\begin{aligned}
u_{i}= & u_{1} \cos \left(\theta_{i}-\theta_{1}\right)+v_{1} \sin \left(\theta_{i}-\theta_{1}\right) \\
& +\dot{\theta}_{1} \frac{d_{1}}{2} \sin \left(\theta_{i}-\theta_{1}\right) \\
& +\sum_{k=2}^{i-1} \dot{\theta}_{k} d_{k} \sin \left(\theta_{i}-\theta_{k}\right) \\
v_{i}= & -u_{1} \sin \left(\theta_{i}-\theta_{1}\right)+v_{1} \cos \left(\theta_{i}-\theta_{1}\right) \\
& +\dot{\theta}_{1} \frac{d_{1}}{2} \cos \left(\theta_{i}-\theta_{1}\right) \\
& +\sum_{k=2}^{i-1} \dot{\theta}_{k} d_{k} \cos \left(\theta_{i}-\theta_{k}\right)+\dot{\theta}_{i} \frac{d_{i}}{2}
\end{aligned}
$$

with $i=2 \ldots N$, leads the extraction of the matrix $\beta$, expressing the relation between the configuration and shape variables.

$$
\dot{s}=\beta \dot{q}
$$

The matrix $\beta$ can be easily computed with the following intermediate relations :

$$
\begin{gathered}
\dot{s}=\beta_{\theta}\left[u_{1} v_{1} \dot{\theta}_{1} \dot{\theta}_{2} \ldots \dot{\theta}_{3}\right]^{T} \\
{\left[u_{1} v_{1} \dot{\theta}_{1} \dot{\theta}_{2} \ldots \dot{\theta}_{3}\right]^{T}=T_{\theta}^{\phi} \dot{q}} \\
\beta=\beta_{\theta} T_{\theta}^{\phi}
\end{gathered}
$$

where

$$
\beta_{\theta}=\left[\begin{array}{ccccccc}
c_{1}^{2} & s_{1}^{2} & \frac{d_{1}}{2} s_{1}^{2} & 0 & 0 & \ldots & 0 \\
-s_{1}^{2} & c_{1}^{2} & \frac{d_{1}}{2} c_{1}^{2} & \frac{d_{2}}{2} & 0 & \ldots & 0 \\
c_{1}^{3} & s_{1}^{3} & \frac{d_{1}}{2} s_{1}^{3} & d_{2} s_{2}^{3} & 0 & \ldots & 0 \\
-s_{1}^{3} & c_{1}^{3} & \frac{d_{1}}{2} c_{1}^{3} & d_{2} c_{2}^{3} & \frac{d_{3}}{2} & \ldots & 0 \\
\ldots & \ldots & \ldots & \ldots & \ldots & \ldots & \ldots \\
c_{1}^{N} & s_{1}^{N} & \frac{d_{1}}{2} s_{1}^{N} & d_{2} s_{2}^{N} & d_{3} s_{3}^{N} & \ldots & 0 \\
-s_{1}^{N} & c_{1}^{N} & \frac{d_{1}}{2} c_{1}^{N} & d_{2} s_{2}^{N} & d_{3} s_{3}^{N} & \ldots & \frac{d_{N}}{2}
\end{array}\right]
$$

with $c_{k}^{i}=\cos \left(\theta_{i}-\theta_{k}\right), s_{k}^{i}=\sin \left(\theta_{i}-\theta_{k}\right)$ and

$$
T_{\theta}^{\phi}=\left[\begin{array}{ccccccc}
1 & 0 & 0 & 0 & 0 & \ldots & 0 \\
0 & 1 & 0 & 0 & 0 & \ldots & 0 \\
0 & 0 & 1 & 0 & 0 & \ldots & 0 \\
0 & 0 & 1 & 1 & 0 & \ldots & 0 \\
\ldots & \ldots & \ldots & \ldots & \ldots & \ldots & \ldots \\
0 & 0 & 1 & 1 & 1 & \ldots & 0 \\
0 & 0 & 1 & 1 & 1 & \ldots & 1
\end{array}\right]
$$


The Lagrangian is then rewritten.

$$
L=\frac{1}{2} \dot{q}^{T}\left(M^{1}+\beta^{T} M^{2} \beta\right) \dot{q}
$$

The dynamic model is derived using

$$
F^{e x t}=\frac{d}{d t} \frac{\partial L}{\partial \dot{q}}-\frac{\partial L}{\partial q}
$$

to obtain

$$
F^{e x t}=\left(M^{1}+\beta^{T} M^{2} \beta\right) \ddot{q}+\left(\beta^{T} M^{2} \dot{\beta}\right) \dot{q}
$$

And the expression for $F^{e x t}=F^{1}+\beta^{T} F^{2}$ yields

$$
\left(M^{1}+\beta^{T} M^{2} \beta\right) \ddot{q}=F^{1}+\beta^{T} F^{2}-\left(\beta^{T} M^{2} \dot{\beta}\right) \dot{q}
$$

where $F^{1}=\left(F_{1}^{u}, F_{1}^{v}, 0, \tau_{2}, \ldots, \tau_{N}\right)$ are the forces that act in the dimensions defined by the configuration variable $q$, and $F^{2}=\left(F_{2}^{u}, F_{2}^{v}, \ldots, F_{N}^{u}, F_{N}^{v}\right)$ contains forces that act in the dimensions defined by the dependent variable $s . \Gamma=$ $\left(\tau_{2}, \ldots, \tau_{N}\right)$ is the vector of control torques.

\section{Control Design}

The control problem is decoupled in 3 sub problems : the thrust generation, the heading control and the combination with a path following requirement.

\section{A. Thrust Control}

The thrust is obtained with a periodic signal propagating along the system body. This reference signal is called 'gait'. A classic approach consists in imposing to the join actuators a sinusoidal based reference of the form : $\phi_{i}=A_{i} \sin \left(w_{i} t+\psi_{i}\right)$ as in [8]. The problem is to adjust the gait parameters $A_{i}, w_{i}$ and $\psi_{i}$ to obtain an efficient thrust generation. Note the work of W. Saintval [4] on an optimization method to evaluate these gait parameters.

We are proposing to use a different approach in the gait generation. The gait we are proposing is not explicitly timedependent and controls the local curvature of the system. This has two advantages.

- The fact that our gait does not explicitly consider the time variable leads to an autonomous system (if the desired velocity is not time dependent), and allows for the use of mathematical tool reserved for autonomous systems.

- Controlling the local curvature is a further step to mimic the behavior of fishes which use a baro-receptors line, deployed along their body, that allows them to locally control the quality of the flow.

Let $s_{i}$ the curvilinear coordinate of the articulation $i$, along the system body; $s_{1}$ denotes the curvilinear position of the head and $s_{N+1}$ is the position of the extremity of the tail. The initial values for $s_{i}$ are : $\left.s_{1}\right|_{t=0}=0,\left.s_{2}\right|_{t=0}=d_{1}$, $\left.s_{3}\right|_{t=0}=d_{1}+d_{2}, \ldots,\left.s_{N+1}\right|_{t=0}=\sum_{k=1}^{N} d_{k}$. The parameterized desired curvature along the system body is expressed as $C^{R}(s)=A \sin (w s)$. The discretization on each system articulation yields :

$$
C_{i}^{R}=A \sin \left(w s_{i}\right)
$$

Let $C_{i}^{E}$ the system curvature of the articulation $i$.

$$
C_{i}^{E}=\frac{\theta_{i}-\theta_{i-1}}{d_{i}+d_{i+1}}=\frac{\phi_{i}}{d_{i}+d_{i+1}}
$$

The control objective is to drive the system curvature $C_{i}^{E}$ to the reference $C_{i}^{R}$, for $i=2, \ldots N$.

\section{1) Kinematic Reference Definition:}

Let $V_{1}=\frac{1}{2} \sum_{i=2}^{N}\left(C_{i}^{R}-c_{i}^{E}\right)^{2}$ be a Lyapunov candidate. Straighforward computation shows that the choice

$$
\dot{C}_{i}^{E}=\dot{C}_{i}^{R}+K_{1}\left(C_{i}^{R}-C_{i}^{E}\right), i=2 \ldots N
$$

yields $\dot{V}_{1}=-K_{1} \sum_{i=2}^{N}\left(C_{i}^{R}-C_{i}^{E}\right)^{2} \leq 0$. Let $C_{i}^{K i n}$ be the kinematic reference for the dynamic control

$$
\dot{C}_{i}^{K i n}=\dot{C}_{i}^{R}+K_{1}\left(C_{i}^{R}-C_{i}^{E}\right), i=2 \ldots N
$$

\section{2) Dynamic Control Design:}

Let $\left.V_{2}=\frac{1}{2} \sum_{i=2}^{N}\left(\dot{C}_{i}^{K i n}-\dot{C}_{i}^{E}\right)^{2}\right]$ be a Lyapunov candidate. The derivation of $V_{2}$ shows that the choice

$$
\ddot{C}_{i}^{E}=\ddot{C}_{i}^{K i n}+K_{2}\left(\dot{C}_{i}^{K i n}-\dot{C}_{i}^{E}\right)
$$

yields $\dot{V}_{2} \leq 0$. Let $C_{i}^{D y n}$ be the dynamic reference.

$$
\ddot{C}_{i}^{D y n}=\ddot{C}_{i}^{K i n}+K_{2}\left(\dot{C}_{i}^{K i n}-\dot{C}_{i}^{E}\right)
$$

This yields to a desired joint acceleration

$$
\ddot{\phi}_{i}^{R}=\left(d_{i}+d_{i-1}\right) \ddot{C}_{i}^{D y n}
$$

The dynamical model of the equation (2) is rewritten

$$
\ddot{q}=\mathcal{M}\left(F^{1}+\mathcal{B}\right)
$$

where $\mathcal{M}=\left(M^{1}+\beta^{T} M^{2} \beta\right)^{-1}$ and $\mathcal{B}=\beta^{T} F^{2}-\left(\beta^{T} M^{2} \dot{\beta}\right) \dot{q}$. The matrices $\mathcal{M}$ and $\mathcal{B}$ are decomposed in:

$$
\begin{aligned}
& \mathcal{M}=\left[\begin{array}{c|c}
\mathcal{M}_{1} & \mathcal{M}_{2} \\
(3 \times 3) & (3 \times(N-1)) \\
\hline \mathcal{M}_{3} & \mathcal{M}_{4} \\
((N-1) \times 3) & ((N-1) \times(N-1))
\end{array}\right] \\
& \mathcal{B}=\left[\begin{array}{c}
\mathcal{B}_{1} \\
\frac{(3 \times 1)}{\mathcal{B}_{2}} \\
((N-1) \times 1)
\end{array}\right]
\end{aligned}
$$

The control is then written as :

$$
\Gamma=\mathcal{M}_{4}^{-1}\left(\ddot{\phi}^{R}-\mathcal{M}_{3}\left(f_{1}+\mathcal{B}_{1}\right)\right)-\mathcal{B}_{2}
$$

where $f_{1}=\left[F_{1}^{u} F_{1}^{v} 0\right]^{T}$.

This control imposes the system to 'slide' on the sinusoidal curvature reference with a curvilinear velocity $\dot{s}$. 
